\title{
Research emphasis and collaboration in Africa
}

\author{
Anastassios Pouris ${ }^{1}$ and Yuh-Shan $\mathrm{Ho}^{2, *}$ \\ 1. Institute for Technological Innovation, University of Pretoria, Pretoria, South Africa \\ 2.Trend Research Centre, Asia University, Taichung 41354, Taiwan \\ *Corresponding author: e-mail: ysho@asia.edu.tw
}

\section{Abstract}

Scientific co-authorship of African researchers has become a fashionable topic in the recent scientometric literature. Researchers are investigating the effects, modes, dynamics and motives of collaboration in a continental research system which is in an embryonic stage and in different stages of development from country to country. In this article we attempt to provide some additional evidence by examining both patterns of collaboration at country and continental levels and the scientific disciplines emphasised. Our findings indicate that the continent's research emphasises medical and natural resources disciplines to the detriment of disciplines supporting knowledge based economies and societies. Furthermore, we identify that the collaborative patterns in Africa are substantial higher than in the rest of the world. A number of questions related to research collaboration and its effects are raised.

\section{Keywords}

Africa; Collaboration; Research; Co-authorship; Scientometrics

\section{Introduction}

Research collaboration is a sociological phenomenon that is receiving the attention of researchers and governments internationally (Yeung et al. 2005). Researchers are investigating the effects, modes, dynamics and motives of collaboration, while governments utilise research collaboration as a policy instrument for technology transfer from universities and research councils to industry, for knowledge transfer from abroad, as a means to improve diplomatic relations with other countries by creating goodwill, and to gain political capital (Wagner et al. 2002a). Researchers collaborate with each other for various reasons. This can be to improve their visibility and recognition (Narin et al. 1991), to utilise expensive equipment that is not under their control (Meadows and O'Connor 1971; Schubert and Sooryamoorthy 2010), or to acquire expertise and new ideas (Beaver and Rosen 1978) needed for research.

In the policy domain, scientific collaboration has become an important component of science, technology and innovation policy internationally, with substantial resources being allocated by governments for this objective. Wagner et al. (2002b) estimated that the USA was spending US\$3.3 billion in the mid-1990s on international research collaboration. 
Similarly, other developed countries were spending substantial amounts as a percentage of their gross domestic product (Wagner et al. 2001). Russell (1995) and Wagner et al. (2001) have suggested that international collaboration is replacing other models as the preferred method of building scientific capacity in developing countries.

While investigations identify the benefits to be derived from collaboration (at least in the currency of science i.e. citations), this collaboration is not without debate related to the risks and benefits of such activities. Arguments expressed include the concern that the spending on international collaboration is not always to the benefit of the paying country and that critical technologies and key knowledge for competitiveness are given away to competitors. Additional concerns have been voiced that collaborative agreements are subordinate to the interests of science and technology to strategic or political ends. Similarly, in the academic domain, researchers have argued that collaboration may be an endogenous self-perpetuating outcome of science, with substantial costs and no commensurate benefits (Jones et al. 2008).

An issue that has received attention and is of importance in the context of Africa is the dependency of the size of collaboration on the size of the scientific community. Narin et al. (1991) found that international co-authorship is higher for scientifically small countries. They argued that scientists in scientifically small countries have far more scientists outside their country with whom to cooperate and far fewer inside their country than scientists in much larger scientific countries do. The argument appeared to be that the collaborative effort is initiated by researchers in small countries who cannot find collaborators.

However, Melin (1999) concluded that "the results indicate that the situation is much more complex than that large country researchers collaborate less internationally than small countries as their scientists more easily can find their partners within the national borders than in smaller countries." Similarly, Boshoff (2009) identified that north-south collaboration takes place in a particular format with the south collaborator basically assisting in fieldwork and data collection. In other words the developed countries' researchers seek collaboration in order to access data and conditions available in the developing countries.

Historically studies on research collaboration were focused on or used data from industrialised countries. More recently, a number of such studies include developing countries in general (Arunachalam and Viswanathan 2008) and African countries (Boshoff 2009; Sooryamoorthy 2009) in particular. Sooryamoorthy (2009) investigated the collaboration patterns of South African researchers and Boshoff (2010) identified the collaborative patterns in the Southern African development community (SADC) countries. Onyancha and Maluleka (2011) found out that knowledge production through collaborative research among sub-Saharan African countries is minimal. Schubert and Sooryamoorthy (2010) showed that "a theory of scientific collaboration building on the notion of marginality and centre-periphery can explain many facets of South African-German collaboration, where South Africa is a semi-peripheral region, a centre for the periphery, and a periphery for the centre". 
In the context of African collaboration it should be emphasised that scientometric studies in general and collaboration studies in particular are in an embryonic stage on the African continent. Even South Africa, which is the major producer of research publications on the continent, produces few publications in the field of scientometrics (Pouris 2012).

In this article, the authors use co-authorship analysis to identify the state of research collaboration on the African continent. The questions they attempt to answer are as follows:

- Which scientific disciplines are emphasised in Africa?

- How did research collaboration evolve in Africa during the period 2007-2011?

- Who are the main research partners of African countries?

- Are the patterns of collaboration (extended and disciplinary) in Africa similar to those in the rest of the world?

- How do the various African countries perform in terms of collaboration?

- Which are the main African institutions that are actively engaged in collaboration?

This article goes on to outline the approach the researchers followed and the data sources used. It follows a results and a discussion section and the article ends with conclusions.

\section{Data sources and methodology}

Since Price and Beaver (1966) used co-authorship as an indicator of research collaboration, it has become an established method, and a multitude of articles have investigated this phenomenon. The approach has gained popularity, even though it is not without criticism (Katz and Martin 1997; Laudel 2002). In this article we use co-authorship analysis in order to identify the collaborative patterns of African researchers.

Data used in this study was retrieved from the Thomson Reuters Web of Science. Again it should be mentioned that bibliometrics in general and the use of particular databases in particular may have their own shortcomings (Roland 2007; Leydesdorff 2008). For this investigation it may be relevant that African countries may publish their research in local journals and languages which are not covered by the Web of Science. However, we should emphasize that in South Africa the government and the university authorities take actions and provide incentives so the researchers publish in the web of Science indexed journals.

The online version of the Science Citation Index Expanded (SCl-Expanded) was accessed on 18 March 2013. In this study, all journal articles in the SCl-Expanded version that were published by authors on the African continent were selected and analysed in order to identify publishing institutions and countries, and to classify articles as collaborative and single-authored publications. The database was searched using the keywords "Algeria", "Angola”, "Benin", "Botswana”, "Burkina Faso", "Burundi”, "Cameroon”, "Cape Verde”, "Cent Afr Republ", "Chad", "Comoros", “Congo", "Cote Ivoire”, "Dem Rep Congo", "Djibouti", "Egypt", “Equat Guinea”, "Eritrea”, "Ethiopia”, "Gabon”, "Gambia”, "Ghana”, "Guinea”, "Guinea Bissau”, "Kenya”, "Lesotho", "Liberia”, "Libya”, "Madagascar", "Malawi”, "Mali", "Mauritania”, "Mauritius", "Morocco", "Mozambique”, "Namibia”, "Niger", "Nigeria", "Rwanda", "Sao Tome and Prin", "Senegal”, "Seychelles", "Sierra Leone”, "Somalia”, "South Africa", "South Sudan", "Sudan", "Swaziland”, "Tanzania”, "Togo", 
"Tunisia", "Uganda", "Western Sahara", "Zambia", "Zimbabwe" and "Zaire" in the address field.

The researchers limited the publication year to between 2007 and 2011, and articles were the only document type considered. Document information such as names of authors, title, year of publication, source journal publishing the articles, contact address, research areas in the Web of Science subject category were downloaded using Microsoft excel. Additional coding was performed manually in order to identify the institutional address of the collaborators.

Affiliations originating from England, Scotland, Northern Ireland and Wales were reclassified as being from the UK (United Kingdom). "Dem Rep Congo" and "Zaire" were reclassified as being from the Democratic Republic of the Congo. Collaboration type was determined by the affiliation of the authors, where the term "internationally collaborative publication" (ICP) was assigned to those articles that were co-authored by researchers from at least two countries. The term "inter-institutionally collaborative publication" was assigned to those articles that were co-authored by researchers from at least two institutions ( $\mathrm{Li}$ and Ho 2008). The term "institutional independent article" was assigned to articles where the researchers' affiliation was from the same institution. Similarly, the term "African collaborative publication" (ACP) was assigned to articles if authors' affiliations were from different countries on the African continent. The term "outside African continent collaborative publication" (OCP) was assigned if articles were co-authored by authors from Africa and authors from countries outside the African continent. The identified articles were further allocated to the Web of Science subject categories. The journal citation reports (JCR) of 2011 indexes 8,336 journals, classified across 176 web of science categories.

\section{Results and discussion}

\section{Language of publication}

A total of 112,576 articles were identified. In order to confirm that these articles were published by authors on the African continent, the researchers further examined the affiliations of authors, and excluded articles that were not published by authors in countries on the African continent, which had been accidentally included in the original set. A total of 111,877 articles published by authors in African countries between 2007 and 2011 were therefore analysed. These articles were published in 17 languages, with the majority of them (97\%) being published in English. The non-English language articles were published in French (3,396 articles), German (51), Spanish (39), Portuguese (16), Italian (6), Korean (5), Chinese (4), Russian (3), Arabic (2), Croatian (2), Dutch (2), Japanese (2), Turkish (2), Hungarian (1), Polish (1), and Welsh (1). The importance of the French language was not surprising, since a number of countries on the African continent were French colonies (Chuang et al. 2011).

\section{Output in research areas}

Figure 1 shows the distribution of research articles in the various countries. The African publications were allocated to various research areas as categorized in the web of science 
categories. Table 1 shows the research areas emphasised in the continent, while Table 2 shows the areas that are underemphasised. These tables also show the number of world publications in the particular fields, the number of African publications, the African share and the activity indices. The activity index characterises the relative research effort a country/region devotes to a given field. It is defined as the country's share in the world's publication output in the given field, divided by the share of the country/region in the world's publication output in all science fields. An index above one means that the region overemphasises the particular field above the world average. An index below one indicates an effort below the world average. An index of one indicates that the region's effort in the particular field corresponds precisely to the world average.

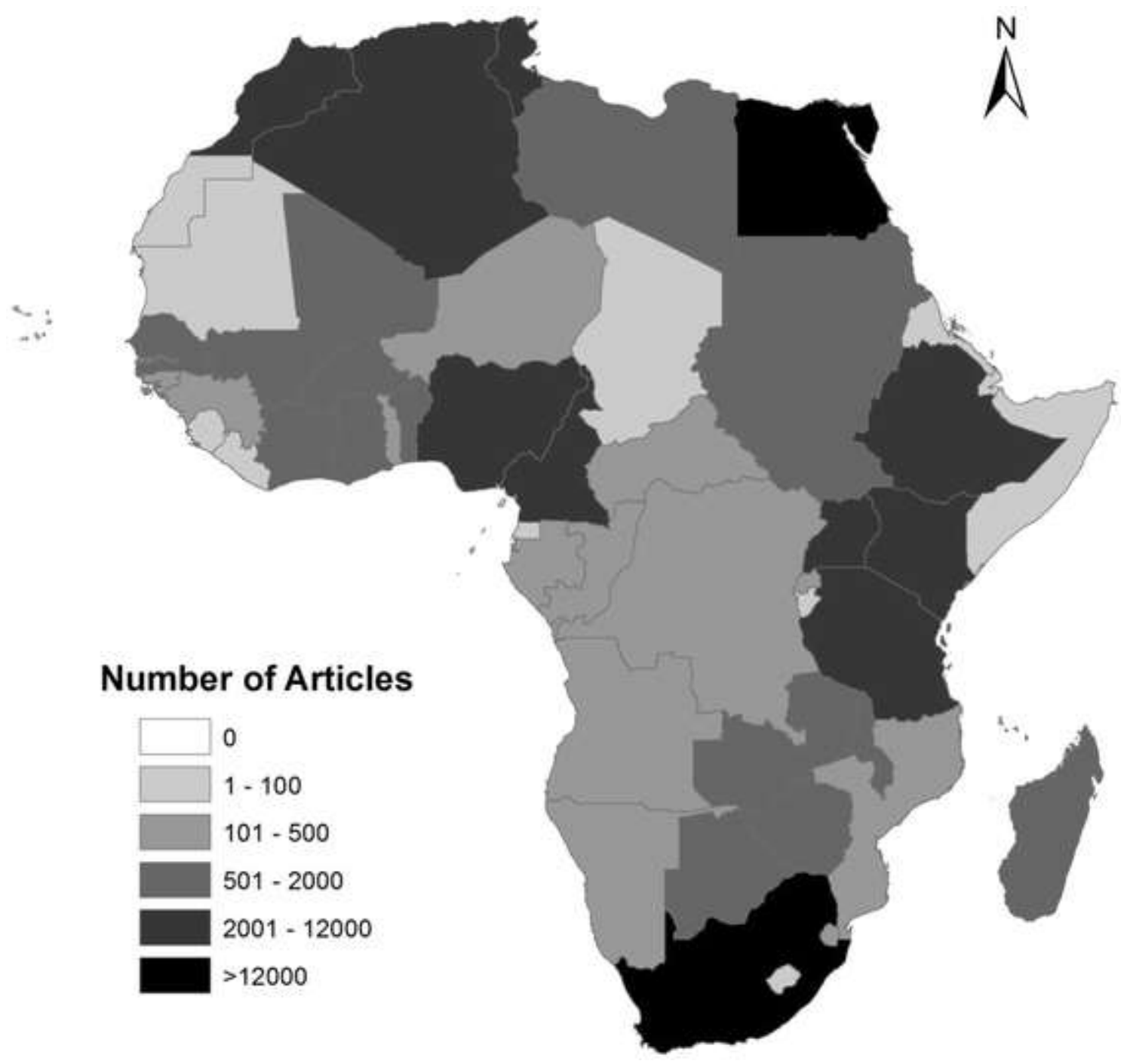

Fig. 1. Distribution of articles published 
Table 1. Emphasised African research areas

\begin{tabular}{|c|c|c|c|c|}
\hline Research areas & The world & Africa & $\%$ & Activity index \\
\hline Tropical medicine & 12,378 & 3,083 & 25 & 13 \\
\hline Parasitology & 19,632 & 2,457 & 13 & 6.5 \\
\hline Infectious diseases & 48,237 & 4,380 & 9.1 & 4.6 \\
\hline Literature & 804 & 62 & 7.7 & 3.9 \\
\hline Integrative and complementary medicine & 8,458 & 634 & 7.5 & 3.8 \\
\hline Anthropology & 3,930 & 251 & 6.4 & 3.2 \\
\hline Public, environmental and occupational health & 73,289 & 4,181 & 5.7 & 2.9 \\
\hline Biodiversity and conservation & 16,695 & 953 & 5.7 & 2.9 \\
\hline Water resources & 45,377 & 2,531 & 5.6 & 2.8 \\
\hline Entomology & 27,051 & 1,503 & 5.6 & 2.8 \\
\hline Virology & 28,696 & 1,587 & 5.5 & 2.8 \\
\hline Mineralogy & 10,054 & 531 & 5.3 & 2.7 \\
\hline Agriculture & 133,673 & 6,105 & 4.6 & 2.3 \\
\hline Plant Sciences & 83,768 & 3,848 & 4.6 & 2.3 \\
\hline Mining and mineral processing & 10,927 & 504 & 4.6 & 2.3 \\
\hline Mycology & 7,778 & 341 & 4.4 & 2.2 \\
\hline Archaeology & 2,377 & 102 & 4.3 & 2.2 \\
\hline Food science and technology & 81,540 & 3,390 & 4.2 & 2.1 \\
\hline Zoology & 56,458 & 2,279 & 4.0 & 2.0 \\
\hline Immunology & 89,896 & 3,394 & 3.8 & 1.9 \\
\hline Medical laboratory technology & 13,189 & 503 & 3.8 & 1.9 \\
\hline Microbiology & 81,321 & 2,981 & 3.7 & 1.9 \\
\hline Veterinary sciences & 67,767 & 2,495 & 3.7 & 1.9 \\
\hline Evolutionary biology & 24,591 & 899 & 3.7 & 1.9 \\
\hline Biotechnology and applied microbiology & 108,945 & 3,924 & 3.6 & 1.8 \\
\hline Forestry & 19,401 & 698 & 3.6 & 1.8 \\
\hline Paleontology & 11,064 & 389 & 3.5 & 1.8 \\
\hline Environmental sciences and ecology & 196,654 & 6,768 & 3.4 & 1.7 \\
\hline Thermodynamics & 30,091 & 1,012 & 3.4 & 1.7 \\
\hline Anatomy and morphology & 8,338 & 280 & 3.4 & 1.7 \\
\hline Geology & 86,994 & 2,801 & 3.2 & 1.6 \\
\hline Life sciences and biomedicine-other topics & 39,673 & 1,259 & 3.2 & 1.6 \\
\hline Demography & 62 & 2 & 3.2 & 1.6 \\
\hline Women's studies & 936 & 29 & 3.1 & 1.6 \\
\hline Marine and freshwater biology & 52,464 & 1,575 & 3.0 & 1.5 \\
\hline Crystallography & 51,400 & 1,546 & 3.0 & 1.5 \\
\hline Obstetrics and gynecology & 46,896 & 1,400 & 3.0 & 1.5 \\
\hline Pharmacology and pharmacy & 165,444 & 4,853 & 2.9 & 1.5 \\
\hline Sociology & 593 & 17 & 2.9 & 1.5 \\
\hline Nutrition and dietetics & 38,840 & 1,105 & 2.8 & 1.4 \\
\hline
\end{tabular}




\begin{tabular}{|c|c|c|c|c|}
\hline Research areas & The world & Africa & $\%$ & Activity index \\
\hline Medical ethics & 3,404 & 97 & 2.8 & 1.4 \\
\hline General and internal medicine & 100,127 & 2,602 & 2.6 & 1.3 \\
\hline Mechanics & 70,984 & 1,841 & 2.6 & 1.3 \\
\hline Toxicology & 41,595 & 1,061 & 2.6 & 1.3 \\
\hline Energy and fuels & 64,345 & 1,640 & 2.5 & 1.3 \\
\hline Pediatrics & 61,885 & 1,521 & 2.5 & 1.3 \\
\hline Fisheries & 21,795 & 543 & 2.5 & 1.3 \\
\hline Physical geography & 17,587 & 433 & 2.5 & 1.3 \\
\hline Mathematics & 234,623 & 5,611 & 2.4 & 1.2 \\
\hline Respiratory system & 33,092 & 789 & 2.4 & 1.2 \\
\hline Ethnic studies & 166 & 4 & 2.4 & 1.2 \\
\hline Polymer science & 73,242 & 1,602 & 2.2 & 1.1 \\
\hline Nuclear science and technology & 44,050 & 987 & 2.2 & 1.1 \\
\hline Meteorology and atmospheric sciences & 45,643 & 951 & 2.1 & 1.1 \\
\hline Reproductive biology & 19,454 & 411 & 2.1 & 1.1 \\
\hline Electrochemistry & 48,073 & 977 & 2.0 & 1.0 \\
\hline Geochemistry and geophysics & 39,154 & 794 & 2.0 & 1.0 \\
\hline Pathology & 33,635 & 671 & 2.0 & 1.0 \\
\hline Remote sensing & 11,176 & 225 & 2.0 & 1.0 \\
\hline Social sciences-other topics & 7,195 & 144 & 2.0 & 1.0 \\
\hline Science and technology-other topics & 180,934 & 3,354 & 1.9 & 1.0 \\
\hline Metallurgy and metallurgical engineering & 74,295 & 1,424 & 1.9 & 1.0 \\
\hline Spectroscopy & 37,743 & 717 & 1.9 & 1.0 \\
\hline Oceanography & 26,790 & 502 & 1.9 & 1.0 \\
\hline Construction and building technology & 19,630 & 372 & 1.9 & 1.0 \\
\hline Imaging science and photographic technology & 8,304 & 155 & 1.9 & 1.0 \\
\hline Legal medicine & 6,576 & 122 & 1.9 & 1.0 \\
\hline
\end{tabular}

Table 2. Under-emphasised African research areas

\begin{tabular}{|l|c|c|c|c||}
\hline \multicolumn{1}{|c|}{ Research areas } & The world & Africa & \% & Activity index \\
\hline \hline Chemistry & 623,271 & 11,528 & 1.8 & 0.9 \\
\hline \hline Urology and nephrology & 45,024 & 827 & 1.8 & 0.9 \\
\hline \hline Health care sciences and services & 30,713 & 546 & 1.8 & 0.9 \\
\hline Dermatology & 27,952 & 516 & 1.8 & 0.9 \\
\hline \hline Biomedical social sciences & 4,024 & 74 & 1.8 & 0.9 \\
\hline Engineering & 558,483 & 9,459 & 1.7 & 0.9 \\
\hline \hline Dentistry, oral surgery and medicine & 36,438 & 622 & 1.7 & 0.9 \\
\hline \hline Automation and control systems & 31,445 & 530 & 1.7 & 0.9 \\
\hline \hline Government and law & 874 & 15 & 1.7 & 0.9 \\
\hline \hline Materials science & 361,943 & 5,863 & 1.6 & 0.8 \\
\hline \hline Genetics and heredity & 79,815 & 1,315 & 1.6 & 0.8 \\
\hline
\end{tabular}




\begin{tabular}{|c|c|c|c|c|}
\hline Research areas & The world & Africa & $\%$ & Activity indes \\
\hline Astronomy and astrophysics & 77,633 & 1,232 & 1.6 & 0.8 \\
\hline Art & 546 & 9 & 1.6 & 0.8 \\
\hline Family studies & 187 & 3 & 1.6 & 0.8 \\
\hline Otorhinolaryngology & 23,397 & 360 & 1.5 & 0.8 \\
\hline Business and economics & 22,197 & 333 & 1.5 & 0.8 \\
\hline Rheumatology & 18,640 & 285 & 1.5 & 0.8 \\
\hline Allergy & 9,165 & 141 & 1.5 & 0.8 \\
\hline Geography & 1,811 & 28 & 1.5 & 0.8 \\
\hline Physics & 593,653 & 8,326 & 1.4 & 0.7 \\
\hline Education and educational research & 15,159 & 214 & 1.4 & 0.7 \\
\hline Urban studies & 976 & 14 & 1.4 & 0.7 \\
\hline Surgery & 139,516 & 1,832 & 1.3 & 0.7 \\
\hline Research and experimental medicine & 59,840 & 778 & 1.3 & 0.7 \\
\hline Instruments and instrumentation & 55,371 & 706 & 1.3 & 0.7 \\
\hline Ophthalmology & 38,176 & 515 & 1.3 & 0.7 \\
\hline Operations research and management science & 33,648 & 423 & 1.3 & 0.7 \\
\hline Behavioral sciences & 24,654 & 312 & 1.3 & 0.7 \\
\hline Biochemistry and molecular biology & 279,571 & 3,313 & 1.2 & 0.6 \\
\hline Computer science & 170,265 & 2,091 & 1.2 & 0.6 \\
\hline Endocrinology and metabolism & 67,517 & 786 & 1.2 & 0.6 \\
\hline Hematology & 44,092 & 547 & 1.2 & 0.6 \\
\hline Sport sciences & 32,560 & 394 & 1.2 & 0.6 \\
\hline Information science and library science & 6,058 & 71 & 1.2 & 0.6 \\
\hline Microscopy & 4,343 & 53 & 1.2 & 0.6 \\
\hline Communication & 674 & 8 & 1.2 & 0.6 \\
\hline Architecture & 489 & 6 & 1.2 & 0.6 \\
\hline Optics & 103,674 & 1,126 & 1.1 & 0.6 \\
\hline Psychiatry & 55,756 & 640 & 1.1 & 0.6 \\
\hline Telecommunications & 48,460 & 511 & 1.1 & 0.6 \\
\hline Orthopedics & 41,713 & 463 & 1.1 & 0.6 \\
\hline Mathematical and computational biology & 23,777 & 261 & 1.1 & 0.6 \\
\hline Social Issues & 1,905 & 21 & 1.1 & 0.6 \\
\hline Arts and humanities-other topics & 87 & 1 & 1.1 & 0.6 \\
\hline $\begin{array}{l}\text { Radiology, nuclear medicine and medical } \\
\text { imaging }\end{array}$ & 75,168 & 760 & 1.0 & 0.5 \\
\hline Anesthesiology & 17,247 & 173 & 1.0 & 0.5 \\
\hline Emergency medicine & 12,628 & 120 & 1.0 & 0.5 \\
\hline Substance abuse & 7,750 & 80 & 1.0 & 0.5 \\
\hline Physiology & 48,460 & 453 & 0.93 & 0.5 \\
\hline Nursing & 26,005 & 242 & 0.93 & 0.5 \\
\hline Acoustics & 19,411 & 180 & 0.93 & 0.5 \\
\hline Cardiovascular system and cardiology & 106,760 & 964 & 0.90 & 0.5 \\
\hline
\end{tabular}




\begin{tabular}{|l|c|c|c|c||}
\hline \multicolumn{1}{|c|}{ Research areas } & The world & Africa & \% & Activity index \\
\hline \hline Rehabilitation & 16,268 & 141 & 0.87 & 0.4 \\
\hline \hline Gastroenterology and hepatology & 49,125 & 420 & 0.85 & 0.4 \\
\hline \hline Transportation & 13,596 & 115 & 0.85 & 0.4 \\
\hline \hline Transplantation & 22,339 & 179 & 0.80 & 0.4 \\
\hline \hline Oncology & 123,272 & 959 & 0.78 & 0.4 \\
\hline Neurosciences and neurology & 216,089 & 1,459 & 0.68 & 0.3 \\
\hline \hline Robotics & 5,615 & 38 & 0.68 & 0.3 \\
\hline \hline Audiology and speech-language pathology & 7,006 & 45 & 0.64 & 0.3 \\
\hline \hline Biophysics & 55,541 & 340 & 0.61 & 0.3 \\
\hline \hline History and philosophy of science & 6,749 & 41 & 0.61 & 0.3 \\
\hline \hline Cell biology & 101,734 & 561 & 0.55 & 0.3 \\
\hline \hline Psychology & 40,044 & 221 & 0.55 & 0.3 \\
\hline \hline Medical informatics & 8,938 & 48 & 0.54 & 0.3 \\
\hline \hline Mathematical methods in social sciences & 6,290 & 32 & 0.51 & 0.3 \\
\hline \hline Linguistics & 1,454 & 7 & 0.48 & 0.2 \\
\hline \hline Developmental biology & 18,645 & 88 & 0.47 & 0.2 \\
\hline \hline Philosophy & 1,384 & 6 & 0.43 & 0.2 \\
\hline \hline Geriatrics and gerontology & 16,030 & 63 & 0.39 & 0.2 \\
\hline \hline Music & 375 & 1 & 0.27 & 0.1 \\
\hline \hline
\end{tabular}

Table 1 shows that the most emphasised research fields are those of tropical medicine (12.5 times bigger than that expected from the scientific size of Africa), parasitology (6.5 times bigger) and infectious diseases (4.6 times bigger). The list of emphasised research areas are dominated by medical and natural resources fields (biodiversity, water resources, entomology, mining, etc.).

Table 2 shows the research areas that are underemphasised in Africa. The list includes areas underpinning modern technologies and economies (i.e. engineering, physics, chemistry, materials science, instrumentation and similar research areas). In contrast it should be mentioned that China, which probably has the most directed scientific system, emphasises engineering, physics and chemistry (National Science Board 2010). The obvious question is why Africa does not follow international examples? It is interesting and debatable to consider whether Africa's needs are served best by the current emphasis. The argument is that the small research community and activity on the continent will not be able to resolve current scientific challenges, such as the HIV/AIDS pandemic. If the regional capacity is not able to provide a scientific or technological solution to a challenge, overemphasis to particular disciplines will not be fruitful. Similarly, while internationally the effort is to develop high technology industries based on brain power, African countries ignore these trends. Hence, the argument can be developed that it may be preferable to move away from expensive fields like medicine and focus on wealth-creating disciplines that may require less investment and may be easier to be diffused in the economy and society. 


\section{Characteristics of collaborative publication outputs}

Figure 2 shows the growth in single-country articles and internationally collaborative articles from the African continent. During the five-year period, the number of articles increased by $50 \%$. The single-country articles increased by $35 \%$, while the internationally collaborative articles grew by $66 \%$-almost twice the growth of the single-country articles. It is interesting to compare the share of internationally collaborative articles from Africa ( $54 \%$ of 111,877 articles) with those in other countries during the period 2007-2011. A comparison of publications and collaborations in the top 20 prolific countries in the world is shown in Table 3. A total of 5,114,346 articles were published in SCl-Expanded version over the same period. The BRIC members, which include Brazil (26\%), Russia (33\%), India (20\%), and China (23\%), had relatively similar percentages of internationally collaborative articles. Higher percentages could be found in the G7 countries, including the USA (33\%), Germany (51 \%), Japan (26\%), the UK (54\%), France (52\%), Italy (44\%), and Canada (49\%).

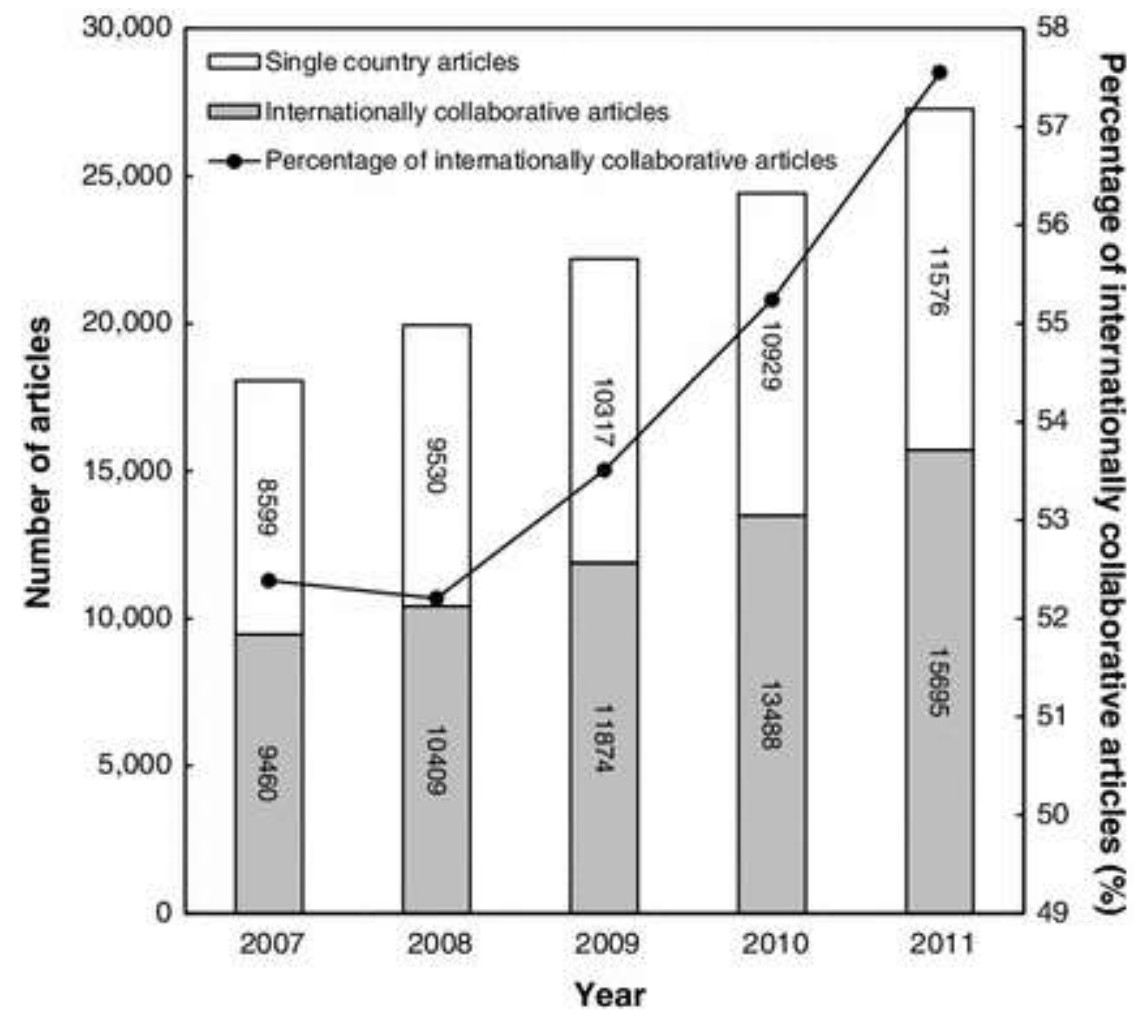

Fig. 2. Growth in African collaboration: 2007-2011 
Table 3. Collaborative Patterns of the top 20 productive countries (2007-2011 in SCl-Expanded)

\begin{tabular}{|c|c|c|c|}
\hline Country & $\begin{array}{c}\text { Total articles (\% of the } \\
\text { world) }\end{array}$ & IP (\% of a country) & ICP (\% of a country) \\
\hline USA & $1,377,409(27)$ & $921,697(67)$ & $455,712(33)$ \\
\hline China & $609,146(12)$ & $469,411(77)$ & $139,735(23)$ \\
\hline Germany & $386,163(7.6)$ & $188,830(49)$ & $197,333(51)$ \\
\hline Japan & $363,394(7.1)$ & $269,136(74)$ & $94,258(26)$ \\
\hline UK & $362,217(7.1)$ & $168,412(46)$ & $193,805(54)$ \\
\hline France & $283,128(5.5)$ & $134,793(48)$ & $148,335(52)$ \\
\hline Italy & $226,000(4.4)$ & $126,788(56)$ & $99,212(44)$ \\
\hline Canada & $224,989(4.4)$ & $115,556(51)$ & $109,433(49)$ \\
\hline India & $190,070(3.7)$ & $151,389(80)$ & $38,681(20)$ \\
\hline Spain & $188,464(3.7)$ & $103,978(55)$ & $84,486(45)$ \\
\hline South Korea & $180,047(3.5)$ & $131,826(73)$ & $48,221(27)$ \\
\hline Australia & $153,574(3.0)$ & $78,101(51)$ & $75,473(49)$ \\
\hline Brazil & $140,722(2.8)$ & $103,731(74)$ & $36,991(26)$ \\
\hline Russia & $131,586(2.6)$ & $88,485(67)$ & $43,101(33)$ \\
\hline Netherlands & $121,934(2.4)$ & $53,964(44)$ & $67,970(56)$ \\
\hline Taiwan & $109,105(2.1)$ & $85,455(78)$ & $23,650(22)$ \\
\hline Turkey & $97,418(1.9)$ & $81,078(83)$ & $16,340(17)$ \\
\hline Switzerland & 94,797 (1.9) & $31,210(33)$ & $63,587(67)$ \\
\hline Poland & $88,638(1.7)$ & $58,232(66)$ & $30,406(34)$ \\
\hline Sweden & $85,693(1.7)$ & $35,250(41)$ & $50,443(59)$ \\
\hline
\end{tabular}

IP single-country articles; ICP internationally collaborative articles

However, as shown in Table 4, the individual African countries exhibit substantially higher collaboration patterns. Nigeria was the only country with a collaboration rate lower than $50 \%$. Twenty-nine countries published more than $90 \%$ of their articles in collaboration with other countries. It is possible that the division of the continent into 54 countries may be a contributor to the substantial number of collaborative articles but other factors may also affect the apparent pattern.

Table 4. Structure of research collaboration in African countries

\begin{tabular}{|l|c|c|c||c||c||c||}
\hline \multicolumn{1}{|c|}{ Country } & Total articles & IP (\%) & ICP (\%) & OCP (\%) & ACP (\%) & SP (\%) \\
\hline \hline South Africa & 29,473 & $13,743(47)$ & $15,730(53)$ & $14,585(49)$ & $1,145(3.9)$ & $2,493(8.5)$ \\
\hline Egypt & 24,126 & $13,726(57)$ & $10,400(43)$ & $10,247(42)$ & $153(0.63)$ & $3,853(16)$ \\
\hline \hline Tunisia & 11,507 & $5,806(50)$ & $5,701(50)$ & $5,552(48)$ & $149(1.3)$ & $391(3.4)$ \\
\hline \hline Nigeria & 9,664 & $6,887(71)$ & $2,777(29)$ & $2,228(23)$ & $549(5.7)$ & $900(9.3)$ \\
\hline \hline Algeria & 7,391 & $3,025(41)$ & $4,366(59)$ & $4,269(58)$ & $97(1.3)$ & $404(5.5)$ \\
\hline Morocco & 6,153 & $2,447(40)$ & $3,706(60)$ & $3,595(58)$ & $111(1.8)$ & $226(3.7)$ \\
\hline Kenya & 4,480 & $731(16)$ & $3,749(84)$ & $3,483(78)$ & $266(5.9)$ & $97(2.2)$ \\
\hline Cameroon & 2,483 & $518(21)$ & $1,965(79)$ & $1,734(70)$ & $231(9.3)$ & $85(3.4)$ \\
\hline
\end{tabular}




\begin{tabular}{|c|c|c|c|c|c|c|}
\hline Country & Total articles & IP (\%) & ICP (\%) & OCP (\%) & ACP (\%) & SP (\%) \\
\hline Uganda & 2,411 & $373(15)$ & $2,038(85)$ & $1,901(79)$ & $137(5.7)$ & $73(3.0)$ \\
\hline Tanzania & 2,354 & 330 (14) & $2,024(86)$ & $1,904(81)$ & $120(5.1)$ & $86(3.7)$ \\
\hline Ethiopia & 2,350 & $688(29)$ & $1,662(71)$ & $1,545(66)$ & $117(5.0)$ & $156(6.6)$ \\
\hline Ghana & 1,700 & $437(26)$ & $1,263(74)$ & $1,182(70)$ & $81(4.8)$ & $63(3.7)$ \\
\hline Senegal & 1,293 & $204(16)$ & $1,089(84)$ & $985(76)$ & $104(8.0)$ & $22(1.7)$ \\
\hline Sudan & 1,063 & $333(31)$ & $730(69)$ & $688(65)$ & $42(4.0)$ & $49(4.6)$ \\
\hline Malawi & 1,059 & 144 (14) & $915(86)$ & $808(76)$ & $107(10)$ & $27(2.5)$ \\
\hline Burkina Faso & 1,008 & $86(8.5)$ & $922(91)$ & $836(83)$ & $86(8.5)$ & $15(1.5)$ \\
\hline Zimbabwe & 1,007 & $165(16)$ & $842(84)$ & $663(66)$ & $179(18)$ & $40(4.0)$ \\
\hline Cote d Ivoire & 936 & $269(29)$ & $667(71)$ & $632(68)$ & $35(3.7)$ & $15(1.6)$ \\
\hline Benin & 852 & $109(13)$ & $743(87)$ & $661(78)$ & $82(10)$ & $14(1.6)$ \\
\hline Madagascar & 782 & $68(8.7)$ & 714 (91) & $703(90)$ & $11(1.4)$ & $11(1.4)$ \\
\hline Zambia & 739 & $4 \quad 41(5.5)$ & 698 (94) & $658(89)$ & 40 (5.4) & $18(2.4)$ \\
\hline Botswana & 721 & $191(26)$ & $530(74)$ & $392(54)$ & $138(19)$ & $75(10)$ \\
\hline Libya & 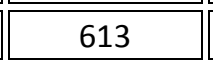 & $179(29)$ & $434(71)$ & $371(61)$ & "63(10) & $53(8.6)$ \\
\hline Mali & 538 & $32(5.9)$ & 506 (94) & $479(89)$ & $27(5.0)$ & $8(1.5)$ \\
\hline Mozambique & 492 & $21(4.3)$ & 471 (96) & 437 (89) & $34(6.9)$ & $8(1.6)$ \\
\hline Gabon & 433 & $16(3.7)$ & 417 (96) & $399(92)$ & $18(4.2)$ & $9(2.1)$ \\
\hline Congo & 3884 & $29(7.6)$ & 355 (92) & $322(84)$ & $333(8.6)$ & $11(2.9)$ \\
\hline Gambia & 384 & $21(5.5)$ & 363 (95) & 357 (93) & 6 (1.6) & $5(1.3)$ \\
\hline Niger & 371 & $28(7.5)$ & 343 (92) & $283(76)$ & 60 (16) & $5(1.3)$ \\
\hline Namibia & 349 & 39 (11) & 310 (89) & $261(75)$ & 49 (14) & $19(5.4)$ \\
\hline Democratic Republic of the Congo & 310 & $20(6.5)$ & $290(94)$ & $276(89)$ & $14(4.5)$ & $5(1.6)$ \\
\hline Rwanda & 275 & $15(5.5)$ & $260(95)$ & $235(85)$ & $25(9.1)$ & $7(2.5)$ \\
\hline Mauritius & 249 & $90(36)$ & $159(64)$ & $149(60)$ & $10(4.0)$ & $17(6.8)$ \\
\hline Togo & 231 & $54(23)$ & $177(77)$ & $154(67)$ & $23(10)$ & $5(2.2)$ \\
\hline Swaziland & 152 & 29 (19) & $123(81)$ & $79(52)$ & $444(29)$ & $14(9.2)$ \\
\hline Angola & 116 & $4(3.4)$ & $112(97)$ & $106(91)$ & $6(5.2)$ & $1(0.86)$ \\
\hline Seychelles & 112 & $4(3.6)$ & $108(96)$ & $106(95)$ & $2(1.8)$ & $3(2.7)$ \\
\hline Guinea Bissau & 111 & $2(1.8)$ & $109(98)$ & $109(98)$ & $0(0)$ & $1(0.90)$ \\
\hline Guinea & 109 & $4(3.7)$ & $105(96)$ & $99(91)$ & $6(5.5)$ & $1(0.92)$ \\
\hline Central African Republic & 105 & $7(6.7)$ & $98(93)$ & $87(83)$ & $11(10)$ & $0(0)$ \\
\hline Mauritania & 86 & $4(4.7)$ & 82 (95) & $57(66)$ & $25(29)$ & $5(5.8)$ \\
\hline Eritrea & 86 & $8(9.3)$ & 78 (91) & $77(90)$ & $1(1.2)$ & $2(2.3)$ \\
\hline Lesotho & 83 & $7(8.4)$ & $76(92)$ & $45(54)$ & $31(37)$ & $2(2.4)$ \\
\hline Sierra Leone & 79 & $8(10)$ & $71(90)$ & $63(80)$ & $8(10)$ & $2(2.5)$ \\
\hline Chad & 73 & $5(6.8)$ & $68(93)$ & $60(82)$ & $8(11)$ & $4(5.5)$ \\
\hline Burundi & 67 & 1 (1.5) & 66 (99) & 64 (96) & $2(3.0)$ & $1(1.5)$ \\
\hline Cape Verde & 30 & $00(0)$ & $30(100)$ & $30(100)$ & $0(0)$ & $00(0)$ \\
\hline Djibouti & 26 & 3 (12) & $23(88)$ & $222(85)$ & 10 & $3(12)$ \\
\hline Liberia & 23 & $0(0)$ & $23(100)$ & $22(96)$ & $1(4.3)$ & $1(4.3)$ \\
\hline
\end{tabular}




\begin{tabular}{|l|c|c|c|c|c|c||}
\hline \hline \multicolumn{1}{|c|}{ Country } & Total articles & IP (\%) & ICP (\%) & OCP (\%) & ACP (\%) & SP (\%) \\
\hline \hline Comoros & 20 & $0(0)$ & $20(100)$ & $16(80)$ & $4(20)$ & $1(5.0)$ \\
\hline \hline Equatorial Guinea & 18 & $0(0)$ & $18(100)$ & $18(100)$ & $0(0)$ & $0(0)$ \\
\hline \hline Somalia & 8 & $0(0)$ & $8(100)$ & $6(75)$ & $2(25)$ & $0(0)$ \\
\hline \hline Sao Tomé and Principe & 7 & $0(0)$ & $7(100)$ & $7(100)$ & $0(0)$ & $0(0)$ \\
\hline \hline Western Sahara & 1 & $0(0)$ & $1(100)$ & $1(100)$ & $0(0)$ & $0(0)$ \\
\hline
\end{tabular}

IP single-country articles; ICP articles published by multiple countries; OCP articles published in collaboration with countries outside the African continent; $A C P$ articles published in collaboration with countries on the African continent only; SP single-author articles; $\%$ percentage of articles in a country

To summarise, on the African continent, internationally collaborative articles grew from 52 to $58 \%$ over the 2007-2011 period. Internationally, articles that list institutions from more than one country, i.e. internationally co-authored articles, also grew dramatically, but only from 10 to $24 \%$ over the 1990-2010 period (National Science Board 2012).

The authors have already referred to the finding that international co-authorship is higher for scientific small countries. However, it is important from a policy perspective to identify the benefits or otherwise of international collaboration on the African continent. Does the African agenda direct the collaborative research agenda or is collaboration directed by international imperatives?

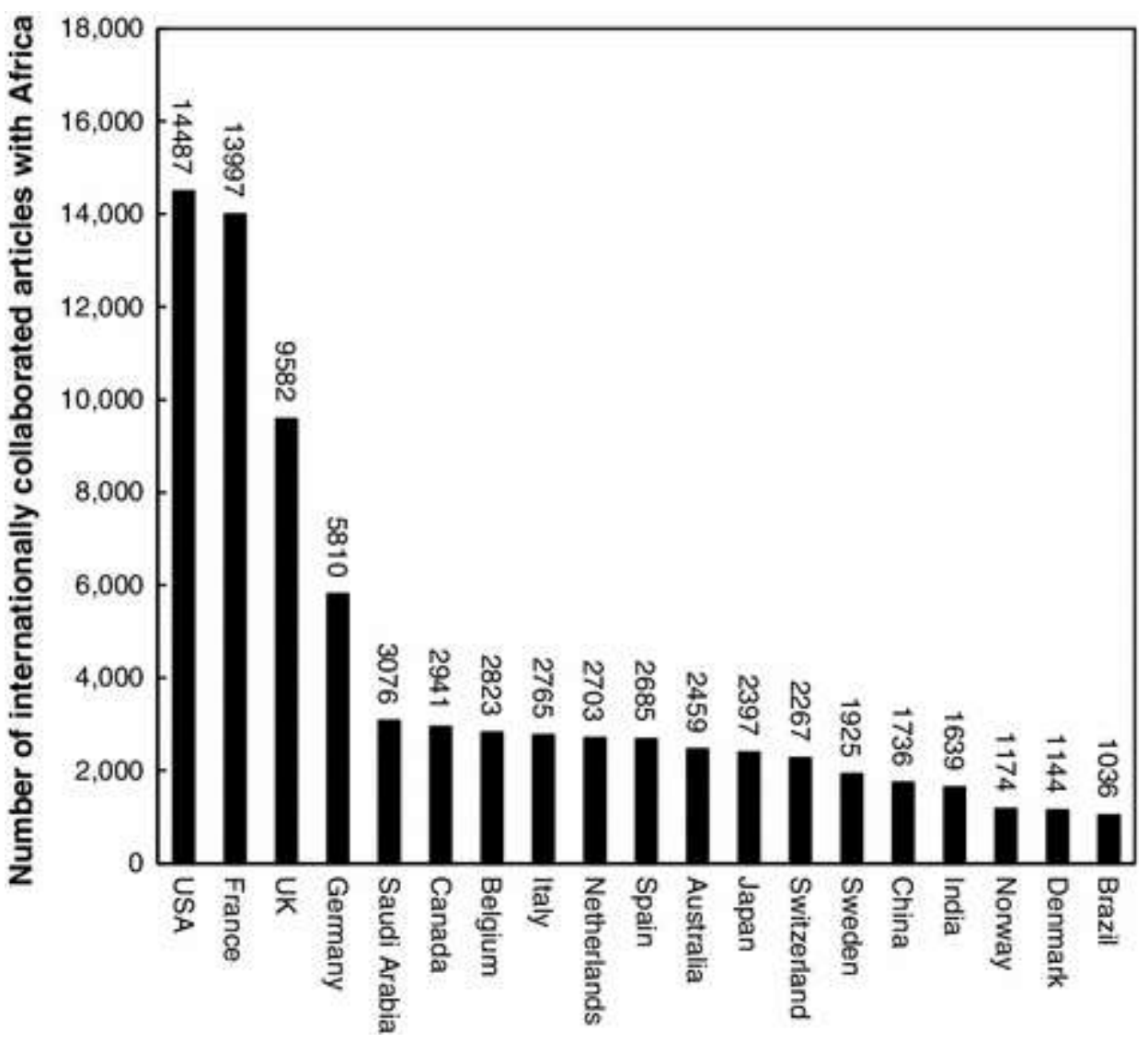

Fig. 3. Main collaborating countries with Africa 2007-2011 
Figure 3 shows the main countries collaborating with Africa. The USA, France and the UK are the main collaborating partners, which produce many more publications with authors from Africa than other countries do. It is important to note that these countries are the most collaborative countries in the world (National Science Board 2012). The three countries (USA, France, and UK) are also the largest funders of research in biosciences, with more emphasis on medicines and agricultural sciences, in Africa.

Table 4 shows the collaborative patterns of individual countries in Africa. The table shows the number of articles produced by individual countries over the 2007-2011 period, the share of articles co-authored internationally (ICP), the share of single-authored articles (SP), the share of articles co-authored with at least one author outside the African continent (OCP) and the share of articles co-authored with authors on the African continent (ACP).

The SP column is informative. Egypt and Botswana had the highest share of single-authored articles (16 and $10 \%$ respectively). The share of single-authored articles is very small (a single-digit number for most countries). As these figures cover all scientific disciplines (those that may need collaboration and those that do not), this can raise the question as to whether there is a scarcity of researchers on the continent that are able to undertake research on their own. The ICP column shows that, with the exception of Nigeria (29\%) and Egypt (43\%), all other countries produce more collaborative articles with co-authors from other countries than with local co-authors.

It is important to note that the number of OCP articles is many times bigger than the ACP articles. What drives researchers, say in Botswana and Zimbabwe, to produce more than $74 \%$ of their collaborative publications outside Africa? South African universities are a few hours away by car. Europe and the USA are a number of hours away by plane. Similarly, why does Egypt collaborate almost exclusively with non-African countries? It may be argued that African collaboration is not driven by local researchers searching for collaborators, but by the availability of resources and interests outside the continent.

Table 5 identifies the most prolific institutions on the African continent and the structure of their publications for the period 2007-2011. Egyptian (9) and South African (7) institutions dominate the list. Nigeria, Uganda, Tunisia and Ethiopia also appear in the list. All institutions have a larger number of inter-institutional collaborative articles than singleinstitution articles.

$S P$ single-author articles; IP single-institution articles; ICP articles published by multiple institutions; OCP articles published in collaboration with institutions outside the African continent; $A C P$ articles published in collaboration with institutions on the African continent only; \% percentage of articles in an institution

It should be emphasised that in South Africa, the funding system of universities - where universities are subsidised by the government according to the number of publications produced by their members of staff (Pouris 1991) -is a disincentive to inter-institutional collaboration. Collaborating institutions have to share the government subsidy. 
Table 5. Most prolific institutions in Africa and their collaborative patterns (2007-2011)

\begin{tabular}{|c|c|c|c|c|c|c|}
\hline Institution & $\begin{array}{c}\text { Total } \\
\text { articles }\end{array}$ & SP (\%) & IP (\%) & ICP (\%) & OCP (\%) & ACP (\%) \\
\hline University of Cape Town, South Africa & 5,454 & $\begin{array}{c}302 \\
(5.5) \\
\end{array}$ & $994(18)$ & $\begin{array}{c}4,460 \\
(82)\end{array}$ & $\begin{array}{c}3,283 \\
(60)\end{array}$ & $\begin{array}{c}107 \\
(2.0)\end{array}$ \\
\hline Cairo university, Egypt & 4,151 & $422(10)$ & $\begin{array}{c}1,212 \\
(29)\end{array}$ & $\begin{array}{c}2,939 \\
(71)\end{array}$ & $\begin{array}{c}1,573 \\
(38)\end{array}$ & $\begin{array}{c}12 \\
(0.29)\end{array}$ \\
\hline $\begin{array}{l}\text { University of the Witwatersrand, South } \\
\text { Africa }\end{array}$ & 3,955 & $\begin{array}{c}364 \\
(9.2) \\
\end{array}$ & $996(25)$ & $\begin{array}{c}2,959 \\
(75)\end{array}$ & $\begin{array}{c}2,080 \\
(53)\end{array}$ & $98(2.5)$ \\
\hline University of Stellenbosch, South Africa & 3,884 & $193(50)$ & $\begin{array}{c}1,030 \\
(27)\end{array}$ & $\begin{array}{c}2,854 \\
(73)\end{array}$ & $\begin{array}{c}1,819 \\
(47)\end{array}$ & $82(2.1)$ \\
\hline University of Pretoria, South Africa & 3,790 & $\begin{array}{c}232 \\
(6.1)\end{array}$ & $\begin{array}{c}1,158 \\
(31)\end{array}$ & $\begin{array}{c}2,632 \\
(69)\end{array}$ & $\begin{array}{c}1,557 \\
(41)\end{array}$ & $\begin{array}{l}184 \\
(4.9)\end{array}$ \\
\hline University of KwaZulu-Natal, South Africa & 3,413 & $\begin{array}{l}290 \\
(8.5) \\
\end{array}$ & $\begin{array}{c}1,011 \\
(30)\end{array}$ & $\begin{array}{c}2,402 \\
(70)\end{array}$ & $\begin{array}{c}1,654 \\
(48) \\
\end{array}$ & $\begin{array}{c}134 \\
(3.9) \\
\end{array}$ \\
\hline Ain Shams university, Egypt & 2,664 & $369(14)$ & $869(33)$ & $\begin{array}{c}1,795 \\
(67) \\
\end{array}$ & $938(35)$ & $\begin{array}{c}15 \\
(0.56) \\
\end{array}$ \\
\hline National research centre, Egypt & 2,659 & $\begin{array}{c}141 \\
(5.3)\end{array}$ & $917(34)$ & $\begin{array}{c}1,742 \\
(66)\end{array}$ & $824(31)$ & $27(1.0)$ \\
\hline Mansoura university, Egypt & 1,926 & $278(14)$ & $823(43)$ & $\begin{array}{c}1,103 \\
(57) \\
\end{array}$ & $723(38)$ & $5(0.26)$ \\
\hline University of Alexandria, Egypt & 1,852 & $315(17)$ & $657(35)$ & $\begin{array}{c}1,195 \\
(65) \\
\end{array}$ & $844(46)$ & $23(1.2)$ \\
\hline University Ibadan, Nigeria & 1,537 & $93(6.1)$ & $447(29)$ & $\begin{array}{c}1,090 \\
(71)\end{array}$ & $396(26)$ & $\begin{array}{l}105 \\
(6.8) \\
\end{array}$ \\
\hline Makerere university, Uganda & 1,347 & $40(3.0)$ & $151(11)$ & $\begin{array}{c}1,196 \\
(89) \\
\end{array}$ & $\begin{array}{c}1,014 \\
(75)\end{array}$ & $64(4.8)$ \\
\hline Assiut university, Egypt & 1,267 & $173(14)$ & $410(32)$ & $857(68)$ & $591(47)$ & \begin{tabular}{|c|}
1 \\
$(0.079)$ \\
\end{tabular} \\
\hline Rhodes university, South Africa & 1,204 & $71(5.9)$ & $416(35)$ & $788(65)$ & $501(42)$ & $61(5.1)$ \\
\hline Suez canal university, Egypt & 1,165 & $140(12)$ & $246(21)$ & $919(79)$ & $702(60)$ & $8(0.69)$ \\
\hline Faculté des Sciences de Tunis, Tunisia & 1,135 & $26(2.3)$ & $230(20)$ & $905(80)$ & $580(51)$ & $23(2.0)$ \\
\hline Al Azhar university, Egypt & 1,062 & $110(10)$ & $220(21)$ & $842(79)$ & $429(40)$ & $2(0.19)$ \\
\hline Zagazig university, Egypt & 1,061 & $152(14)$ & $378(36)$ & $683(64)$ & $411(39)$ & $9(0.85)$ \\
\hline University of Johannesburg, South Africa & 1,015 & $80(7.9)$ & $331(33)$ & $684(67)$ & $448(44)$ & $21(2.1)$ \\
\hline University of Addis Ababa, Ethiopia & 936 & $56(6.0)$ & $178(19)$ & $758(81)$ & $540(58)$ & $28(3.0)$ \\
\hline
\end{tabular}

The high share of inter-institutional collaborative articles from South African universities indicates that the forces promoting inter-institutional collaboration are stronger than the adverse impact of the funding mode. It should be mentioned that at other universitiessuch as the National Taiwan University (21\%) and Peking University in China (31 \%) (Wang et al. 2011)-internationally collaborative articles make up a lower percentage of the total number of articles. 


\section{Conclusions}

This article set as its objective the identification of the co-authorship patterns of research on the African continent (as they are manifested in the Thomson Reuters indexed journals) and the elaboration of the findings. The authors identified, from a policy perspective, the importance of assessing the benefits or otherwise of international collaboration on the African continent. While the majority of the international literature considers scientific collaboration to be beneficial for both partners, there is no scarcity of the opposite arguments.

For example, arguments have been expressed that the USA may lose out due to the Asian strength, which may be fuelled by globalisation trends. Similarly, in the African context, it has been argued that South Africa spends considerable research effort in the field of HIV/AIDS; well above what is expected from its relative scientific size, and it is doubtful that the HIV/AIDS epidemic can be resolved by South African research alone, without the support of the rest of the world. This emphasis may need further assessment (Pouris and Pouris 2011). Scientific small countries, because of their scientific limitations, have to be particularly attentive to their research priorities in order to optimise their developmental goals.

The above argument is further supported by the identified disciplinary emphasis of Africa's research. Africa's research emphasises natural resources and medical fields. While it can be argued that this emphasis is underlined by the resources available on the continent and the diseases present, it may be argued that these priorities may not necessarily be the best options for the continent's developmental objectives. It should be mentioned that Africa countries have limited research prioritisation mechanisms, and any embryonic efforts in this domain are based on the immediate needs of the existing activities, and not on the most achievable and beneficial efforts for the future when the research outputs will materialise.

In this context, the Asian research priorities are informative. Why is the research focus of China and other Asian countries on engineering, physics and chemistry (disciplines supporting knowledge-based societies) while Africa focuses on medical and natural resources?

Identification of the research outputs of the African countries and their related collaborative patterns shows that the continent suffers from subcritical research systems and collaboration dominance. Single-author articles appear to be on the verge of extinction on the continent. It may be argued that this is the effect of the foreign funding sources which favour group of researchers and not individual researchers. The revealed structure raises a number of policy concerns. Should Africa's science and development not be better served by the creation of regional research and innovation systems (that is aiming to create an African Research Union)? How do the high dependencies on non-African collaboration affect the continent's research evolution and priorities? Is African research individualism and inspiration stifled by excessive collaboration? 


\section{Acknowledgments}

The authors wish to thank Professor J. Mugabe and an anonymous referee for comments and suggestions of a previous version of the article. The normal caveat applies.

\section{References}

Arunachalam, S., \& Viswanathan, B. (2008). South-south cooperation: The case of indo-chinese collaboration in scientific research. Current Science, 95(3), 311-313.

Beaver, D., \& Rosen, R. (1978). Studies in scientific collaboration: Part I-professional origins of scientific co-authorship. Scientometrics, 1(1), 65-84.

Boshoff, N. (2009). Neo-colonialism and research collaboration in central Africa. Scientometrics, $81(2), 413-434$.

Boshoff, N. (2010). South-south research collaboration of countries in the southern African development community (SADC). Scientometrics, 84(2), 481-503.

Chuang, K. Y., Chuang, Y. C., Ho, M., \& Ho, Y. S. (2011). Bibliometric analysis of public health research in Africa: The overall trend and regional comparisons. South African Journal of Science, 107(5/6), 5459.

Jones, B. F., Wuchty, S., \& Uzzi, B. (2008). Multi-university research teams: Shifting impact, geography, and stratification in science. Science, 322(5905), 1259-1262.

Katz, J. S., \& Martin, B. R. (1997). What is research collaboration? Research Policy, 26(1), 1-18.

Laudel, G. (2002). What do we measure by co-authorships? Research Evaluation, 11(1), 3-15.

Leydesdorff, L. (2008). Caveats for the use of citation indicators in research and journal evaluation. Journal of the American Society for Information Science and Technology, 59(2), 278-287.

Li, Z., \& Ho, Y. S. (2008). Use of citation per publication as an indicator to evaluate contingent valuation research. Scientometrics, 75(1), 97-110.

Meadows, A. J., \& O'Connor, J. G. (1971). Bibliographical statistics as a guide to growth points in science. Science Studies, 1(1), 95-99.

Melin, G. (1999). Impact of national size on research collaboration: A comparison between northern European and American universities. Scientometrics, 46(1), 161-170.

Narin, F., Stevens, K., \& Whitlow, E. S. (1991). Scientific co-operation in Europe and the citation of multinationally authored papers. Scientometrics, 21(3), 313-323.

National Science Board. (2010). Science and engineering indicators 2010. Arlington: National Science Foundation (NSB 10-01). 
National Science Board. (2012). Science and Engineering Indicators Digest 2012. Arlington: National Science Foundation (NSB 12-02).

Onyancha, O. B., \& Maluleka, J. R. (2011). Knowledge production through collaborative research in sub-Saharan Africa: How much do countries contribute to each other's knowledge output and citation impact? Scientometrics, 87(2), 315-336.

Pouris, A. (1991). Effects of funding policies on research publications in South Africa. South African Journal of Science, 87(3-4), 78-81.

Pouris, A. (2012). Scientometric research in South Africa and successful policy instruments. Scientometrics, 91, 317-325.

Pouris, A., \& Pouris, A. (2011). Scientometrics of a pandemic: HIV/AIDS research in South Africa and the world. Scientometrics, 86, 541-552.

Price, D. J. D., \& Beaver, D. D. (1966). Collaboration in an invisible college. American Psychologist, 21(11), 1011-1018.

Roland, E. (2007). On the use and abuse of bibliometric indicators: A critique of Hix's global ranking of political departments. European Political Science, 6(3), 306-314.

Russell, J. M. (1995). The increasing role of international cooperation in science and technology research in Mexico. Scientometrics, 34(1), 45-61.

Schubert, T., \& Sooryamoorthy, R. (2010). Can the centre-periphery model explain patterns of international scientific collaboration among threshold and industrialised countries? The case of South Africa and Germany. Scientometrics, 83(1), 181-203.

Sooryamoorthy, R. (2009). Collaboration and publication: How collaborative are scientists in South Africa? Scientometrics, 80(2), 419-439.

Wagner, C. S., Brahmakulam, I. T., Jackson, B. A., Wong, A., \& Yoda, T. (2001). Science and technology collaboration: Building capacity in developing countries?. Santa Monica: Rand.

Wagner, C. S., Brahmakulam, I. T., Peterson, D. J., Staheli, L., \& Wong, A. (2002a). U.S. government funding for science and technology cooperation with Russia. Santa Monica: Rand.

Wagner, C. S., Staheli, L., Silberglitt, R., Wong, A., \& Kadtke, J. (2002b). Linking effectively: Learning lessons from successful collaboration in science and technology. Santa Monica: Rand.

Wang, M. H., Fu, H. Z., \& Ho, Y. S. (2011). Comparison of universities' scientific performance using bibliometric indicators. Malaysian Journal of Library and Information Science, 16(2), 1-19.

Yeung, Y. Y., Liu, T. C. Y., \& Ng, P. H. (2005). A social network analysis of research collaboration in physics education. American Journal of Physics, 73(2), 145-150. 